\title{
Le mouvement associatif garibaldien en France
} de 1945 au début des années 2000 : un internationalisme ancré dans une culture politique franco-italienne

Il movimento associativo garibaldino in Francia tra il 1945 e l'inizio degli anni 2000: un internazionalismo che ha le sue radici in una cultura politica franco-italiana

Jérémie Dubois

\section{OpenEdition}

\section{Journals}

Édition électronique

URL : http://journals.openedition.org/cei/5298

DOI : 10.4000/cei.5298

ISSN : 2260-779X

Éditeur

UGA Éditions/Université Grenoble Alpes

Édition imprimée

ISBN : 978-2-37747-076-1

ISSN : 1770-9571

\section{Référence électronique}

Jérémie Dubois, «Le mouvement associatif garibaldien en France de 1945 au début des années 2000 : un internationalisme ancré dans une culture politique franco-italienne », Cahiers d'études italiennes [En ligne], 28 | 2019, mis en ligne le 15 février 2019, consulté le 27 mars 2021. URL http://journals.openedition.org/cei/5298; DOI : https://doi.org/10.4000/cei.5298

Ce document a été généré automatiquement le 27 mars 2021 


\title{
Le mouvement associatif
} garibaldien en France de 1945 au début des années 2000 : un internationalisme ancré dans une culture politique francoitalienne

\author{
Il movimento associativo garibaldino in Francia tra il 1945 e l'inizio degli \\ anni 2000: un internazionalismo che ha le sue radici in una cultura politica \\ franco-italiana
}

Jérémie Dubois

1 Né à Nice en 1807, mort sur l'île italienne de Caprera le 2 juin 1882, Giuseppe Garibaldi a donné naissance, de son vivant puis de façon posthume à un "mythe politique ${ }^{1}$ " durable, multiforme et transnational. Pour avoir combattu lors des principales étapes $\mathrm{du}$ processus national unitaire italien au $\mathrm{XIX}^{\mathrm{e}}$ siècle, Garibaldi occupe une place singulière dans la culture mémorielle italienne ${ }^{2}$. Son nom a cependant aussi servi de vecteur de mobilisation associative dans d'autres pays où il avait combattu, et en particulier en France. La particularité de ce mythe tient au fait qu'il dépasse largement Garibaldi comme individu: les garibaldiens de France se réfèrent autant aux engagements du combattant italien qu'à ceux de personnes qui se sont réclamées de lui ou ont mené des combats dans lesquels ils perçoivent une continuité avec les siens.

2 Si des recherches approfondies ont déjà été menées, en particulier par Hubert Heyriès, sur l'action des garibaldiens en France lors de la Première Guerre mondiale et dans l'entre-deux-guerres ${ }^{3}$, ce mouvement demeure moins étudié pour la période qui a suivi la Libération ${ }^{4}$, même si plusieurs articles ainsi qu'un documentaire en ont souligné l'originalité ${ }^{5}$. Les formes de sociabilités spécifiquement «garibaldiennes » en France 
après la Seconde Guerre mondiale représentent un aspect révélateur de certains traits de la culture italienne en France au $\mathrm{xx}^{\mathrm{e}}$ siècle. Étudier l'Association nationale des garibaldiens de France, dont le siège est à Paris ${ }^{6}$, amène à analyser un ensemble de pratiques sociales et un corpus de valeurs politiques et morales, portées et redéfinies dans la longue durée par les acteurs de ce mouvement, en prenant en compte leurs trajectoires familiales, leurs engagements militants et leurs statuts professionnels. Le destin de cette association éclaire également le rapport des institutions françaises à un univers socio-culturellement lié à l'émigration italienne.

Plusieurs types de sources permettent de comprendre cette culture politique militante singulière, tout à la fois franco-italienne et promotrice de valeurs internationalistes. Nous nous appuierons en particulier sur les archives déposées à la Bibliothèque de documentation internationale contemporaine de Nanterre à la suite du décès de Darno Maffini, qui présida durant trente-neuf ans, entre 1958 et 1997, l'Association nationale des garibaldiens de France ${ }^{7}$. Ces fonds ont été croisés pour l'écriture de cet article avec des témoignages recueillis auprès de membres de l'association, ainsi qu'avec des archives propres de l'association, auxquelles le président Philippe Guistinati a bien voulu nous donner accès.

4 Nous montrerons d'abord comment l'association a évolué dans le temps, entre 1945 et le début des années 2000, avant de nous pencher sur les membres de l'association, sur leurs actions et sur le financement de la structure. Enfin, nous soulignerons que la commémoration, activité importante pour les garibaldiens, participe pour eux d'un travail d'interprétation de l'histoire, mais aussi d'un engagement qui mêle prises de positions et revendications.

\section{Des honneurs républicains à la dissolution forcée}

\subsection{Un groupe valorisé dans le contexte de la Libération}

$5 \quad$ L'un des aspects les plus saisissants de l'histoire du mouvement associatif garibaldien depuis 1945 porte sur les cinq premières années de la période: la structure passe en effet durant cet intervalle des honneurs républicains à la dissolution administrative. Dès le printemps 1945, le colonel Xavier Derfner, ancien combattant de l'Argonne, travaille à la réorganisation d'un groupement des garibaldiens de France ${ }^{8}$. L'une de ses premières grandes réalisations d'après-guerre est un "festival garibaldien ", sorte de soirée de gala qui eut lieu le 16 juin 1945 au théâtre des Champs-Élysées à Paris. Ce festival était organisé «au profit des prisonniers, déportés politiques et des œuvres d'assistance aux Garibaldiens qui ont souffert pour la Liberté ${ }^{~}$. Derfner indique dans ses Mémoires que ce festival fut précédé d'une campagne de publicité à la radio, dans la presse parisienne et par de grandes affiches dans les stations de métro. À ces démarches s'ajoutent une reconnaissance institutionnelle italienne et française. Derfner souligne que le festival garibaldien a été placé sous la présidence d'honneur de l'ambassadeur d'Italie, Giuseppe Saragat ${ }^{10}$, ce que confirme le programme de cette soirée, conservé dans les archives du théâtre des Champs-Élysées et mis en ligne par celui-ci ${ }^{11}$. Derfner souligne que l'entrée du théâtre était gardée «à droite et à gauche par un détachement de la garde républicaine sabre au clair, mis à notre disposition par la préfecture de police, alternant avec un service de Garibaldiens en chemises rouges avec décorations ${ }^{12} »$. Ces honneurs rendus aux garibaldiens s'expliquent sans doute 
largement par le rôle qu'ils ont joué au moment de la Libération de Paris. Cet événement prend tout son sens lorsqu'il est mis en lien avec la suite des rapports de l'Association des garibaldiens de France avec les autorités françaises et italiennes.

\subsection{Entre guerre froide et législation sur les associations étrangères}

L'association voit d'abord son existence légitimée par un décret du 6 avril 1947. Mais trois ans après, l'année 1950 fut celle de toutes les déconvenues pour les garibaldiens de France. Le 2 juin 1950, ils constatent que l'ambassade d'Italie limite sa participation à l'une de leurs cérémonies ${ }^{13}$. En effet, le président de la fédération garibaldienne d'Italie avait adressé un télégramme à l'ambassadeur d'Italie en France dans lequel il annonçait la dissolution de l'association française. Un deuxième acte allait se jouer en décembre 1950 : le ministre de l'Intérieur, Henri Queuille, décida le 26 décembre 1950 de reporter le décret du 6 avril 1947 autorisant l'association ${ }^{14}$. Il en a découlé une dissolution pure et simple de la structure. Derfner indique qu'un de ses interlocuteurs au sein de la police française lui aurait indiqué confidentiellement que « la cause venait de certaines sections de province, par leur propagande extrémiste ${ }^{15}$ » et que lui n'était pas en mesure de les contenir. Cette réponse rapportée est bien sûr difficile à interpréter. Qu'entendre en effet ici par "propagande extrémiste »? Il faut sans doute inscrire la dissolution par le gouvernement de l'Association des garibaldiens de France dans le cadre de la guerre froide et de la lutte contre les éléments réputés liés au communisme ${ }^{16}$. L'organisation a sans doute été dissoute en vertu de la législation du 12 avril 1939 sur les associations étrangères, qui soumet celles-ci à l'autorisation préalable du ministre de l'Intérieur, autorisation qui peut être retirée à tout moment, par décret ${ }^{17}$. À l'Assemblée nationale, lors de la séance du 6 février 1951, le député communiste et ancien maire de Marseille, Jean Cristofol, proteste avec vigueur contre cette décision de dissolution des Garibaldiens de France, la mettant en parallèle avec la suppression d'autres associations hongroise, polonaise ou roumaine. Il reproche au ministre de l'Intérieur le manque de reconnaissance de la France envers des immigrés et des réfugiés politiques qui ont « fait leur devoir pour la France » pendant les deux guerres mondiales, invoquant notamment les volontaires italiens enterrés au cimetière de Bligny ou les combats de la Résistance ${ }^{18}$. Cette dénonciation de l'ingratitude française envers le sacrifice de garibaldiens peut rappeller, dans un tout autre contexte, le souvenir de la colère du député Victor Hugo qui démissionna avec fracas de l'Assemblée nationale en 1871, par solidarité avec Giuseppe Garibaldi, que les députés réunis à Bordeaux avaient moqué lorsqu'il avait voulu plaider la cause des volontaires ayant combattu avec lui pour la France face à la Prusse en 1870-1871.

\subsection{Une association refondée deux ans après sa dissolution}

7 Le 3 janvier 1952, parut au Journal officiel l'annonce de la création d'une nouvelle association française, "Les Garibaldiens ", dont les statuts furent déposés par "un groupe de garibaldiens naturalisés français ${ }^{19} »$. Deux anciens officiers de l'Argonne en assumèrent successivement la présidence, Marfella de 1952 à 1956, puis Palazzi de 1956 à $1958^{20}$. Un changement de génération s'opère en 1958 lorsque Darno Maffini devient le président du groupement. Né en 1908, Darno Maffini était trop jeune pour combattre lors de la guerre de 1914-1918. Il a en revanche pris part à la Deuxième Guerre mondiale au sein de la Résistance, en particulier lors des combats de la Libération de 
Paris. À cinquante ans, il arrive relativement jeune à la tête de l'association. Il dirige dès lors la structure jusqu'en 1997, tout en restant président honoraire de l'association jusqu'à sa mort en 2002. Il est remplacé par Mario Piscina de 1997 à 2001, date à laquelle lui succède Gelsomino Guistinati, qui reste président jusqu'à sa mort en 2005. Rino Marzani le remplace alors puis, depuis mars 2007, Philippe Guistinati, dirige le mouvement ${ }^{21}$.

\section{2. Être garibaldien en France après 1945 : origines et caractéristiques d'un milieu militant}

\subsection{Qui sont les Garibaldiens de France?}

8 La recherche de renseignements sur les membres de l'association aux différentes époques amène à croiser différentes sources. Une coupure de Paris-Jour, journal possédé par le patron de presse Cino Del Duca, mécène de l'association des Garibaldiens, évoque en février 1968 « 2000 membres actifs » au sein des Garibaldiens de France ${ }^{22}$. L'article oppose ce chiffre aux «30000 membres» que l'association aurait comptés à la Libération. Ces chiffres doivent être considérés avec une grande prudence. Philippe Guistinati évoque plutôt «environ 500 membres en région parisienne dans les années 1960-1970». Il décrit ensuite une baisse des effectifs «liée à l'importance de la mortalité », qui a abouti à un étiage à " une centaine de membres en 2000 ». Depuis, il évoque une remontée sensible des adhésions, « liée en particulier aux effets de la mise en place d'un site internet depuis $2005 »$. En 2007, indique Philippe Guistinati, l'association compte 115 membres $^{23}$.

9 Du point de vue de l'approche sociologique, l'association est essentiellement formée par des personnes issues du milieu des ouvriers, des artisans et des entrepreneurs indépendants ${ }^{24}$. Les professions des présidents successifs en témoignent. Darno Maffini était bottier, et, parmi ses successeurs, Mario Piscina était plâtrier, Gelsomino Guistinati gérait une entreprise de sécurité pour le bâtiment, Rino Marzani était restaurateur. Le président actuel, Philippe Guistinati, anime une entreprise de restauration des structures du bâtiment. La composition sociologique du bureau porte elle aussi la marque de cette culture professionnelle ouvrière et artisanale. Occupant les fonctions de trésorière au sein du bureau national des Garibaldiens en 2008, Anna Ladner a travaillé comme bobineuse dans une entreprise de textile que dirigeait son cousin, Fernando Zanca, qui a lui-même été secrétaire général de l'association aux côtés de Darno Maffini ${ }^{25}$. La diversité des métiers exercés par les animateurs du mouvement garibaldien a servi son fonctionnement: Fernando Zanca mettait à la disposition de l'association un camion et un chauffeur de son entreprise pour assurer la logistique des grands événements ${ }^{26}$. Giorgio Marzano, qui siège en 2008 au bureau national comme « responsable des banquets garibaldiens » a exercé dans les métiers de bouche.

10 Ces données professionnelles peuvent être croisées avec des indications sur les origines nationales : la plupart des figures du mouvement des garibaldiens de France sont issues des différentes vagues de l'immigration italienne en France. Darno Maffini, Gelsomino Guistinati et Fernando Zanca arrivent en France dès avant la Deuxième Guerre mondiale. Le bureau de l'association au début des années 2000 comporte une majorité de personnes d'origine italienne, pour la plupart arrivées en France après 1945, certains ayant été naturalisés français, d'autres ayant gardé la nationalité italienne. 
11 Des renseignements peuvent enfin être fournis sur l'implantation géographique de l'association. Dans les archives Darno Maffini de Nanterre figure un fichier de 1996 utilisé pour annoncer le grand repas annuel des garibaldiens à Montreuil ${ }^{27}$. Ce banquet, payant, commémorant en avril la Libération de l'Italie, rassemble les garibaldiens et leurs amis pour financer l'association. Sur 271 foyers destinataires $\mathrm{du}$ courrier, 84 résident à Paris, soit environ un tiers du total ${ }^{28}$. Le $\mathrm{XI}^{\mathrm{e}}$ arrondissement est surreprésenté, il abrite un quart des Parisiens invités par les garibaldiens. Philippe Guistinati propose deux explications : d'une part, «c'est dans ce secteur que se sont déroulés en 1944 les combats pour la caserne de la place de la République, combats dans lesquels les garibaldiens, et parmi eux Darno Maffini, ont joué un grand rôle ». De plus, «des liens très forts existent entre les Garibaldiens de France et l'association des anciens combattants du XI ${ }^{\mathrm{e}}$ arrondissement. Darno Maffini était membre du bureau de cette association jusqu'à sa mort ». Depuis, les garibaldiens Fernand Batut puis Philippe Guistinati lui ont succédé. Roger Fichtenberg, président de l'Union des associations d'anciens combattants et victimes de guerre du XI $\mathrm{I}^{\mathrm{e}}$ arrondissement, a ainsi lancé la procédure pour que Darno Maffini et les garibaldiens soient honorés par l'attribution d'une place parisienne ${ }^{29}$. La "place Darno Maffini» fut inaugurée dans le $\mathrm{XI}^{\mathrm{e}}$ arrondissement le 27 juin 2013.

12 Hors de Paris, l'ancrage géographique des garibaldiens de France se situe principalement dans le bassin de l'Île-de-France : le département de la Seine-SaintDenis est le mieux représenté avec 102 adresses sur 271, réparties sur 23 communes, au premier rang desquelles figurent Montreuil, Bobigny et Bagnolet $^{30}$.

\subsection{Le calendrier garibaldien en France}

13 L'activité des garibaldiens possède plusieurs dimensions qui ont contribué à permettre à l'association de s'inscrire dans la durée. En premier lieu intervient une activité fédératrice, la permanence du samedi après-midi. Cette rencontre lors de laquelle des membres de l'association se retrouvent pour échanger représente un rituel de sociabilité inscrit dans la longue durée. Certains y participent depuis près de quarante ans. Ces rencontres, qui se tiennent au local des Garibaldiens, au 20 rue des Vinaigriers, permettent non seulement aux garibaldiens de se retrouver, mais encore aux personnes intéressées de les rencontrer.

14 Le calendrier garibaldien est également rythmé par un certain nombre de commémorations annuelles. Les garibaldiens de France honorent par exemple le souvenir des résistants étrangers dirigés par l'Arménien Missak Manouchian, non seulement lors de cérémonies au Mont-Valérien, où ces combattants ont été fusillés, mais aussi au cimetière d'Ivry, où ils ont été inhumés. Chaque 25 février, les garibaldiens de France se retrouvent au cimetière d'Ivry pour un hommage ${ }^{31}$.

15 Un deuxième moment de rassemblement des garibaldiens de France intervient en référence à la Libération de l'Italie, le 25 avril $1945^{32}$. Un rassemblement festif et militant se déroule ainsi régulièrement au printemps. En 1969, le banquet se déroule déjà dans le grand hall du parc Montreau de Montreuil ${ }^{33}$; vingt-sept ans après, en 1996, ce repas se tenait toujours au même endroit. Pour comprendre pourquoi les garibaldiens ont déployé leur principale manifestation annuelle en ce lieu, il faut souligner l'appui apporté par la municipalité de Montreuil, qui prêtait gratuitement la salle $^{34}$. Darno Maffini et le maire communiste de Montreuil, Jean-Pierre Brard, 
échangeaient à ce sujet une correspondance directe ${ }^{35}$. Désormais, toutefois, les garibaldiens organisent cette fête dans leurs locaux de la rue des Vinaigriers.

En novembre, les garibaldiens participent aux côtés des chasseurs alpins italiens à une cérémonie organisée à Bligny, en Champagne, en souvenir des combattants garibaldiens de la Première Guerre mondiale. Novembre est encore le mois où, le jour anniversaire de l'armistice, les garibaldiens honorent leurs disparus en déposant des fleurs devant le monument du cimetière du Père-Lachaise. Lors de ces manifestations, les garibaldiens portent la chemise rouge.

Insister sur plusieurs éléments récurrents du calendrier des garibaldiens ne doit pas masquer l'existence d'évolutions: ainsi, les garibaldiens se sont longtemps rendus chaque année à Bagnoles-de-l'Orne, en souvenir de l'assassinat des frères Rosselli en juin $1937^{36}$. De même, l'hommage annuel à la statue parisienne de Garibaldi dans le XVe arrondissement n'est actuellement plus pratiqué. Toutefois, une réflexion est en cours pour que renaissent ces engagements.

\subsection{Une association inscrite dans une constellation de mouvements}

De nombreux garibaldiens s'avèrent également membres d'autres structures et contribuent à insérer leur association au sein d'une constellation associative. L'association des Garibaldiens, lors de sa reconstitution en 1952, bénéficia du patronage d'une autre structure, Union des engagés volontaires et résistants d'origine étrangère (UGEVRE). Celle-ci contribua à l'élaboration des statuts déposés à la préfecture de Paris. Darno Maffini cumula la présidence des Garibaldiens avec celle de l'UGEVRE et avec la vice-présidence de l'ANACR, l'Association nationale d'anciens combattants et résistants. Parmi les membres du bureau des Garibaldiens, nombreux sont ceux qui exercent ou ont exercé des responsabilités dans d'autres structures, locales ou nationales. Mario Piscina a longtemps présidé l'Association franco-italienne de Bagnolet, tandis que le garibaldien Renato Zuliani a longtemps été aussi le président de l'Association des chasseurs alpins italiens de France, les Alpini de France. Les contacts sont également très forts avec le monde ancien combattant, par le biais des portedrapeaux qui, comme Eugène Locatelli, Giorgio Marzano ou Théodore Lazaridis, participent très fréquemment aux cérémonies quotidiennes destinées à raviver la flamme de l'arc de triomphe ${ }^{37}$. Enfin, un facteur matériel contribue à développer les liens entre les garibaldiens et d'autres associations: dans leur local, ils accueillent certaines associations dont ils se sentent proches, comme « Les amis des combattants en Espagne républicaine ».

\subsection{Mécènes et bienfaiteurs : le rôle de la famille Del Duca}

19 L'association des Garibaldiens a la particularité de posséder son lieu de réunion à Paris. Pour l'expliquer il faut évoquer l'action de leurs mécènes. L'action la plus importante fut la protection apportée au mouvement par l'éditeur italien Cino Del Duca, puis par sa femme Simone Nirouet Del Duca. Ce soutien s'étale sur plus d'un demi-siècle. Dès 1945, Cino Del Duca contribua à combler le déficit né du festival garibaldien évoqué plus haut ${ }^{38}$. En 1967, lors d'une campagne de dons pour l'achat du local du 20 rue des Vinaigriers, Cino Del Duca versa 10000 francs. Toutefois, les Garibaldiens, successivement hébergés depuis 1945 rue de Rivoli, rue Vieille-du-Temple puis rue du 
Paradis, entrent d'abord comme locataires en avril 1967. Après le décès de Cino Del Duca, en 1967, sa veuve, Simone Del Duca, a aidé l'association à acquérir son local en deux étapes : son entreprise, les Éditions Mondiales, consentit d'abord un prêt sans intérêt à l'association, qui devint ainsi propriétaire dès 1978. Dix ans après, en 1988, $\mathrm{M}^{\text {me }}$ Del Duca acheva son geste de mécénat en signant un chèque du même montant par lequel elle a remboursé sur ses fonds propres la dette des garibaldiens envers son entreprise ${ }^{39}$.

Pour comprendre pourquoi la famille Del Duca a tant aidé le mouvement garibaldien, il faut évoquer d'abord la réussite fulgurante de Cino Del Duca après son arrivée en France en $1932^{40}$. Il crée d'abord un hebdomadaire pour enfants, puis en 1947 l'hebdomadaire Nous Deux. Naturalisé français en 1957, il rachète le journal socialiste Franc-Tireur, qui devient sous sa direction Paris-Journal puis Paris-Jour ${ }^{41}$.

21 Entre Garibaldi, les garibaldiens et le grand patron de presse, le lien est d'abord d'ordre familial. La nécrologie de Cino Del Duca publiée dans Le Monde en mai 1967 indique qu'il était « fils d'un artisan engagé volontaire dans la légion garibaldienne qui, en 1871, lutta aux côtés de la France ${ }^{42} »$. Un discours de Darno Maffini précise que Giosuè Del Duca, le père de Cino Del Duca, se battit « dans les rangs des chemises rouges sous le commandement de [...] Garibaldi à Dijon ». Le livre d'hommage que Simone Del Duca a fait publier par André Frossard après la mort de son mari livre des précisions sur le cheminement familial et personnel qui a amené le couple Del Duca à engager une partie de sa fortune au service de la cause garibaldienne ${ }^{43}$. Un portrait de Cino Del Duca en chemise rouge orne toujours le mur du local des garibaldiens de France.

\section{Les engagements des garibaldiens, entre commémorations et prises de position}

Les engagements militants des garibaldiens en France peuvent s'analyser à l'aune de leur lien direct à Garibaldi, de leur regard sur l'histoire et de leurs engagements face aux événements de leurs temps, de 1945 aux années 2000.

Parmi les buts pour lesquels combattent les garibaldiens de France après 1945 figure en premier lieu la défense de la mémoire de Garibaldi. Les garibaldiens s'engagent ainsi pour que des lieux soient consacrés à sa mémoire. À Vaulx-en-Velin, en 1980, le choix de la municipalité de nommer une avenue Garibaldi répond aux demandes de la section de Villeurbanne ${ }^{44}$. Ce travail de valorisation du général italien s'accomplit également lors des anniversaires le concernant. Les archives du fonds Maffini de Nanterre témoignent d'une collaboration étroite entre Anita Garibaldi, descendante du général italien et les garibaldiens. Ainsi, ils préparent ensemble le voyage à l'île de Caprera en 1982 à l'occasion du centenaire de la mort du combattant italien.

Les garibaldiens portent ensuite sur l'histoire un regard d'acteur. Si la mémoire des combattants volontaires de l'Argonne est honorée, la réflexion sur la Deuxième Guerre mondiale apparaît aussi très présente chez eux après 1945. La première de leur revendication possède une dimension pratique et réside en la lutte pour la reconnaissance complète de tous les services de résistance. Mais ils s'insèrent également dans la continuité des combats passés et réclament « la mise hors d'état de nuire des auteurs de crimes de guerre ". Derrière des slogans comme "ni Pétain à 
Douaumont, ni Laval au Panthéon!» existe dans la longue durée une attention très forte portée par les garibaldiens aux discours qui peuvent être élaborés sur l'histoire.

Les garibaldiens prennent également position vis-à-vis du temps présent. En 1972, ils participent activement à des actions menées au sein d'un Comité unitaire antifasciste dont le siège est fixé à leur local de la rue des Vinaigriers. Il s'agit de fédérer différentes structures de l'immigration italienne en France, en lien avec le journal L'Emigrante, pour peser sur la situation politique italienne, jugée alors menacée par une résurgence $\mathrm{du}$ fascisme. Les garibaldiens adoptent aussi des positions liées aux relations internationales : en octobre 1979, ils participent à Rome à une " Rencontre mondiale des anciens combattants pour la paix et le désarmement ». De plus, les allusions aux grands événements de la seconde moitié du vingtième siècle affleurent dans leurs discours. En avril 1990, dans le cadre de la commémoration de la libération de l'Italie, Darno Maffini fit directement allusion à la chute du mur de Berlin :

Aujourd'hui, plus que jamais, les hommes devraient se féliciter de voir s'écrouler un peu partout les murs et les barbelés qui empêchaient les citoyens de circuler. Cette ouverture ne sera plus un obstacle à la liberté, elle devra permettre la construction d'une Europe unie, pacifique, tournée vers le progrès social, en somme l'Europe telle que Giuseppe Garibaldi en avait jeté les bases à l'Assemblée européenne réunie à Genève le siècle dernier ${ }^{45}$.

Idéalisme garibaldien et idéalisme européen semblent ainsi parfois se rejoindre au sein de l'association.

En conclusion, la position des garibaldiens de France dans l'espace public alterne entre reconnaissance, mise à l'écart et difficultés surmontées par l'appui de mouvements associatifs ou de soutiens individuels et institutionnels.

Dans l'ensemble, l'association semble avoir aussi servi, dans la longue durée, de structure d'intégration en France pour des personnes issues de l'immigration italienne. Le mouvement garibaldien semble bien contribuer dans la longue durée à une redéfinition de l'image des Italiens en France, en associant l'Italie à l'idée d'un combat démocratique de portée universelle. Dans l'autre sens, il semble permettre à ceux qui y prennent part de concilier leur attachement à leur patrie d'origine avec leur lien à leur patrie d'adoption.

\section{NOTES}

1. Sur les origines de ce mythe, voir G. Pécout, "Garibaldi sans légende ", L'Histoire, n 156 , juin 1992, p. 8. Sur la vie, les usages et la postérité de Garibaldi, voir L. Riall, Garibaldi, Invention of a Hero, Yale, Yale University Press, 2007 ; P. Milza, Garibaldi, Paris, Fayard, 2012 ; S. Patriarca, "Unmaking the Nation? Uses and Abuses of Garibaldi in Contemporary Italy ", Modern Italy, vol. 15, $\mathrm{n}^{\circ} 4$, 2010, p.467-483. P.F. Giorgetti (dir.), Garibaldi: visione nazionale e prospettiva internazionale, Atti del Convegno Nazionale (Livourne, 31 mai- $1^{\mathrm{er}}$ juin 2007), Pise, Edizioni ETS, 2008 ; J.-Y. Frétigné et P. Pasteur (dir.), Garibaldi: modèle, contre-modèle, Mont-Saint-Aignan, Publications des universités de Rouen et du Havre, 2011 ; J. Grévy, Garibaldi, Paris, Presses de Sciences Po, 2001. Sur la construction du mythe de Garibaldi en France, voir M. Agulhon, «Le 
mythe de Garibaldi en France de 1882 à nos jours ", dans Id., Histoire vagabonde, vol. II, Paris, Gallimard, 1988, p. 85-131.

2. Garibaldi peut à ce titre être considéré comme un "lieu de mémoire " de l'Italie contemporaine. M. Isnenghi, «Garibaldi », dans Id. (dir.), L'Italie par elle-même. Lieux de mémoire italiens de 1848 à nos jours, Paris, Éditions rue d'Ulm, 2006, p. 397-418. Voir aussi la forte présence des références à Garibaldi dans les musées italiens du Risorgimento, comme celui de Rome, ou le nombre des monuments qui furent élevés à son effigie dans des villes et villages de la péninsule. Sur la place de la référence à Garibaldi dans le Livre Cour d'Edmondo De Amicis, voir G. Pécout, «Histoire et pédagogie nationale dans l'Italie contemporaine. Les usages du passé proche dans le Livre Cour de De Amicis", Histoire@Politique. Politique, culture, société, n² 2, septembreoctobre 2007, <www.histoire-politique.fr> (consulté le 10 août 2018).

3. Voir H. Heyriès, Les Garibaldiens de 14 : splendeurs et misères des Chemises rouges en France de la Grande Guerre à la Seconde Guerre mondiale, Nice, Serre éd., 2005 ; J. Grévy, H. Heyriès et C. Maltone, Garibaldi et garibaldiens en France et en Espagne: histoire d'une passion pour la démocratie, Pessac, Presses universitaires de Bordeaux, 2011; N. Violle, "Garibaldiens de l'Argonne, soldats du souvenir et messagers de l'Italie Nouvelle », Italies, $\mathrm{n}^{\circ} 19,2015$, <http://journals.openedition.org/ italies/5217> (consulté le 10 août 2018).

4. Nous avions commencé à explorer cet univers pour le colloque organisé en février 2008 à l'École normale supérieure et à l'Institut culturel italien de Paris par Massimo Baioni, Patrizia Dogliani et Gilles Pécout sur «Garibaldi et les garibaldiens en France: images, héritages et réseaux, du $\mathrm{XIX}^{\mathrm{e}}$ au XXI ${ }^{\mathrm{e}}$ siècle ». Nous remercions Gilles Pécout de nous avoir suggéré l'idée de cette enquête.

5. On se reportera à plusieurs présentations récentes de l'association: B. Hopquin, "Les garibaldiens raniment la flamme ", Le Monde, 9 octobre 2014 ; M. Slapater, "Les Garibaldiens : hier, aujourd'hui et demain ? L'héritage d'un idéaliste hors du commun ", Hommes \& Migrations, n $1317-1318,2017 / 2-3$, p. 124-126 ; le président de l'association, Philippe Guistinati, a accordé en 2010 un entretien à la revue Migrance : « La Commune de Paris est notre point de départ, notre étalon dans l'histoire ", Migrance, $\mathrm{n}^{\circ} 35,1^{\mathrm{er}}$ semestre 2010, La Commune et les étrangers, numéro dirigé par Sylvie Aprile, p. 120-123. Voir aussi le DVD du documentaire de Marco Astolfi, Ciao compagni, qui comporte des entretiens avec Darno Maffini: M. Astolfi, Ciao compagni, Paris, L'Harmattan vidéo, 2007 et l'article de P. Rochette, « Les Chemises rouges commémorent l'Affiche rouge. (FTP-MOI) », Libération, 25 février 1995. L'association des Garibaldiens possède également son propre site : «Les Garibaldiens. Association nationale mémorielle et militante née en 1947 », accessible à l'URL suivant: <www.wmaker.net/lesgaribaldiens/L-association-LESGARIBALDIENS_a6.html?com> (consulté le 25 juillet 2018).

6. Dans le cadre de cet article, nous centrons notre analyse sur les activités de l'Association nationale des garibaldiens de France. Parmi les structures garibaldiennes existant en France, on signalera la fédération de Toulouse et de Midi-Pyrénées, dirigée par Henri Basso, qui est dotée d'un site internet : <http://garibaldienstoulouse.free.fr>.

7. Le résistant Darno Maffini est décédé en 2002 ; ses archives ont été versées en 2005 à la BDIC et comptent plus de 1800 documents.

8. Sur Xavier Derfner, parfois également désigné sous le nom de Saverio Derfner, voir H. Heyriès, Les Garibaldiens de 14 : splendeurs et misères des Chemises rouges en France de la Grande Guerre à la Seconde Guerre mondiale, ouvr. cité, p. 376. Derfner avait déjà joué un rôle dans l'associationnisme garibaldien dans l'entre-deux-guerres, notamment comme vice-président de l'Union des garibaldiens de l'Argonne à un moment où celle-ci était dirigée par Camillo Marabini. Sur les engagements de Marabini, voir N. Violle, «Garibaldiens de l'Argonne, soldats du souvenir et messagers de l'Italie Nouvelle ", Italies, $\mathrm{n}^{\circ}$ 19, 2015, <http://journals.openedition.org/italies/5217> (consulté le 10 août 2018). 
9. Cette formule est tirée de X.Derfner, Mémoires d'un légionnaire garibaldien, Bordeaux, Imprimerie Delmas, 1961, p. 179.

10. Opposant au fascisme, Giuseppe Saragat est une des figures politiques majeures de l'Italie d'après-guerre. Après avoir été ambassadeur d'Italie en France, il fut élu président de l'Assemblée constituante en Italie en juin 1946. Il devait plus tard exercer les fonctions de président de la République italienne de 1964 à 1971.

11. Voir le programme du « Festival Garibaldien », 16 juin 1945, accessible sur le site présentant les archives du théâtre des Champs-Élysées à l'adresse suivante: <www.tce-archives.fr/ document/1282-festival-garibaldien> (consulté le 25 juillet 2018).

12. X. Derfner, Les mémoires d'un légionnaire garibaldien, ouvr. cité, 1961, p. 179-180.

13. Ibid., p. 213.

14. Ibid., p. 214.

15. Ibid., p. 214 et suiv.

16. Sur cette problématique, voir P. Buton, O. Büttner et M. Hastings (dir.), La guerre froide vue d'en bas, Paris, CNRS éditions, 2014.

17. Cette nécessité d'une autorisation préalable pour les associations étrangères est restée en vigueur jusqu'au début des années 1980. Voir à ce sujet J. Guedj, « Associations étrangères et vie politique sous la IV ${ }^{e}$ République ", Parlement[s], Revue d'histoire politique, $\mathrm{n}^{\circ} 27,2018 / 1$, p. 77-99 ; C. Andrieu, G. Le Béguec et D. Tartakowsky (dir.), Associations et champ politique : la loi de 1901 à l'épreuve du siècle, Paris, Publications de la Sorbonne, 2001.

18. Intervention du député communiste Jean Cristofol à l'Assemblée nationale, $2^{\mathrm{e}}$ séance du 6 février 1951, Journal officiel de la République française, débats parlementaires de la $4^{\mathrm{e}}$ République, en ligne sur <http://4e.republique.jo-an.fr/page2/1951_p798.pdf?q=garibaldiens> (consulté le 7 octobre 2018).

19. Voir sur cet épisode : X. Derfner, Les mémoires d'un légionnaire garibaldien, ouvr. cité, p. 214 et suiv.

20. Ces informations figurent dans le texte de présentation du «Fonds Darno Maffini », série «F delta 1873 », BDIC Nanterre, établi par Marc Milicet, 2005.

21. Philippe Guistinati est le fils de l'ancien président Gelsomino Guistinati.

22. "Garibaldi : le héros de toutes les générosités », extrait du journal Paris-Jour, 28 février 1968, p. 11, conservé dans les archives Darno Maffini, Carton 7 F delta 1873 (7) (Documents divers).

23. En 2018, l'association compte désormais environ 80 membres, mais ses activités ont été fortement étendues : le local des Garibaldiens, à Paris, accueille désormais, tout au long de la semaine, de nombreuses activités en lien avec la culture italienne en France et avec d'autres associations mémorielles (Entretien téléphonique avec Philippe Guistinati, président de l'Association des garibaldiens de France, 7 octobre 2018).

24. Cette composition sociologique des responsables de l'association peut-être mise en relation avec la forte tradition de création par des personnes issues de l'émigration italienne en France de petites entreprises en région parisienne; voir M. Martini, Bâtiment en famille: migrations et petite entreprise en banlieue parisienne au $\mathrm{XX}^{e}$ siècle, Paris, CNRS éditions, 2016.

25. Entretien avec Anna Ladner, alors trésorière de l'association, au siège des Garibaldiens, Paris, le 8 février 2007.

26. Idem.

27. «Noms et adresses des membres et amis des Garibaldiens. À convoquer pour Montreuil, février $1996 » .7$ pages. Archives Darno Maffini, BDIC, Carton 4 F delta 1873 (4).

28. Certains noms figurant dans la liste sont rayés et comportent la mention « décédé ».

29. Information communiquée par Philippe Guistinati, président de l'Association des garibaldiens de France.

30. «Noms et adresses des membres et amis des Garibaldiens. À convoquer pour Montreuil, février 1996 ». 7 pages. Archives Darno Maffini, BDIC, Carton 4 F delta 1873 (4). 
31. Voir par exemple l'article cité de Philippe Rochette à propos des commémorations de février 1995, «Les Chemises rouges commémorent l'Affiche rouge. (FTP-MOI)», Libération, 25 février 1995.

32. En Italie, le 25 avril est le jour de la «fête de la Libération », en souvenir de l'ordre d'insurrection générale donné par les résistants en 1945. G. Pécout, « Ce que les Italiens célèbrent en ce "jeudi tricolore" ", Le Monde, 16 mars 2011.

33. Voir le manuscrit du discours prononcé par Darno Maffini, 4 mai 1969 au parc Montreau de Montreuil, BDIC, fonds Darno Maffini, F delta 1873 (1), b.

34. Voir la lettre adressée à Darno Maffini, le 22 janvier 1996, par la responsable du service de gestion des salles de la ville de Montreuil et la convention d'utilisation du grand hall du parc Montreau, 4 rue Babeuf à Montreuil («montant de la location : gratuit »), pour la manifestation organisée le dimanche 21 avril 1996.

35. Lettre de Jean-Pierre Brard, député-maire de Montreuil, datée du 9 avril 1996 à Darno Maffini, président national de l'association «Les Garibaldiens », dossier F delta 1873 (1), b, pochette "Libération italienne, 1965-2000", sous-pochette "Fête de la libération italienne à Montreuil en 1996 », BDIC, fonds Darno Maffini, F delta 1873 (1) b.

36. Sur les frères Rosselli, voir A. Giacone et É. Vial (dir.), I fratelli Rosselli: l'antifascimo e l'esilio, Rome, Carocci, 2011.

37. Voir à ce propos l'article cité de B. Hopquin, "Les garibaldiens raniment la flamme», Le Monde, 9 octobre 2014.

38. Voir le passage évoquant Codari et Del Duca à propos du financement du festival garibaldien dans les Mémoires de Xavier Derfner (X. Derfner, Mémoires d'un légionnaire garibaldien, ouvr. cité, p. 179-181).

39. Copie du chèque de $\mathrm{M}^{\text {me }}$ Simone Del Duca à l'ordre de «l'Association française des résistants et volontaires garibaldiens », daté de Paris, le 7 avril 1988, archives propres de l'association. La photocopie porte l'inscription manuscrite suivante: «Chèque remis par Madame Del Duca pour rembourser l'emprunt concédé pour l'acquisition du local des Garibaldiens. »

40. Né en 1899 à Montedinove, sur l'Adriatique, il dut quitter l'Italie en 1932 en raison de ses opinions antifascistes. «L'éditeur Cino Del Duca est mort à Milan », Le Monde, 25 mai 1967, p. 9.

41. Ibid.

42. Ibid. Nous remercions Thomas Wieder de nous avoir procuré cet article.

43. Dans sa préface à l'ouvrage, André Frossard note : «Compagnon de Garibaldi, Giosuè Del Duca se battit pour la France en 1870. Avec sa barbe fleuve et son nez rouge, il arracha aux Prussiens, sous les murs de Dijon, le seul drapeau pris à l'ennemi durant cette campagne. Cino se souviendra de cet exemple lorsqu'il participera, en 1939, à la création de la nouvelle légion garibaldienne, composée d'Italiens décidés à combattre pour la France. » Voir Cino Del Duca, 1899-1967, s. 1., 1967, p. 11.

44. Voir les documents de la sous-pochette F delta 1873 (2) b, fonds Darno Maffini, BDIC, Nanterre.

45. Discours de Darno Maffini, 29 avril 1990, Archives Darno Maffini, BDIC, Nanterre, pochette Discours, F delta 1873 (1) b. 


\section{RÉSUMÉS}

Le mouvement associatif garibaldien représente un vecteur original de présence et de diffusion de la culture italienne en France. L'Association nationale des garibaldiens de France, dont le siège est à Paris, a été tour à tour valorisée par la République française à la Libération puis interdite dans un contexte de guerre froide avant d'être refondée au début des années 1950. Soutenue par des mécènes comme Simone et Cino Del Duca, l'association fut longtemps présidée par l'ancien résistant Darno Maffini. À partir d'archives et d'entretiens, cet article vise à éclairer l'histoire sociale, intellectuelle et économique de ce mouvement qui promeut une culture italienne démocratique et antifasciste en France.

Il movimento associativo garibaldino è un vettore originale di presenza e di diffusione della cultura italiana in Francia. L'associazione nazionale dei garibaldini di Francia, con sede a Parigi, fu valorizzata dalla Repubblica francese al momento della Liberazione, poi vietata nel contesto della «guerra fredda» e rifondata all'inizio degli anni '50. Sostenuta da mecenati, e in particolare da Simone e Cino Del Duca, il movimento è stato diretto per molti anni da Darno Maffini, che aveva partecipato alla Resistenza e contributo alla liberazione di Parigi. A partire da archivi e interviste, il presente articolo si propone di spiegare la storia sociale, intellettuale e economica di questo movimento, che difende in Francia una cultura italiana democratica e antifascista.

\section{INDEX}

Mots-clés : Garibaldi, associations italiennes, immigration italienne, culture politique, mémoire Parole chiave : Garibaldi, associazione italiane, immigrazione italiane, cultura politica, memoria

\section{AUTEUR}

\section{JÉRÉMIE DUBOIS}

Université de Reims Champagne-Ardenne, CERHiC, EA 2616.

jeremie.dubois@univ-reims.fr

Jérémie Dubois est agrégé d'histoire, maître de conférences en histoire contemporaine à

l'université de Reims Champagne-Ardenne. Ses recherches portent sur l'histoire sociale, culturelle, politique de l'enseignement de l'italien en France entre la fin du XIX siècle et 1940, et sur l'articulation entre l'enseignement des langues et de l'histoire et les relations internationales. 